\title{
Inability of protozoa from the kangaroo forestomach to establish in the rumen of sheep
}

\author{
SK Baker 1,2, BA Dehority 3, NL Chamberlain ${ }^{1}$, DB Purser ${ }^{1}$ \\ 1CSIRO Division of Animal Production, Wembley, WA 6014 ; 2 The University of Western Australia, Nedlands \\ WA 6009, Australia ; ${ }^{O}$ Ohio Agricultural Research and Development Center, Wooster OH 44691-4096, USA
}

In the macropodine forestomach is a diverse microbial population of bacteria, ciliate protozoa and fungi. The ciliate protozoa have not been classified, but morphologically they resemble those in the rumen genera Isotricha and Dasytricha (Hunton, 1982, Honours thesis, Univ West Aust; Dellow et al, 1988, Aust J Zool, 36, 383-395). The bacterial population is morphologically less diverse than that in the rumen ; yet the size structures of the bacterial populations are similar (Baker, 1989, AJAS, 2, 458-459). If this similarity reflects similarities in the trophic structures of the ecosystems, then the ciliate protozoa in the macropodine forestomach may occupy trophic niches similar to those of the ciliate protozoa in the rumen. This study was conducted to determine if ciliate protozoa from the macropodine forestomach would establish in the ovine rumen.

Two groups each of 10 wether sheep were housed in separate rooms. They were fed ad libitum a diet of oaten hay, oaten straw, oat grain, lupin grain and supplementary minerals $(59.4,10.4,14,14,2.2)$, offered once daily. Five sheep in each group were offered the diet either chopped or finely ground. The sheep were housed in individual pens and each was separated from its neighbour by an empty pen. Protozoa were removed from the rumen using alkanate $3 S L 3(3 \mathrm{ml} 10 \%$ alkanate $/ \mathrm{kg}$ live weight, intraruminally). Digesta (ca $200 \mathrm{~g}$ ) combined from the forestomachs of 3 western grey kangaroos (Macropus fuligninosus) was introduced into the rumen of each sheep in one room (Group K) via a rumen cannula. There were $0.50 \times 10^{5}$ ciliates $/ g$ in the inoculum from the kangaroos and they resembled those described by Dellow et al (1988) and Hunton (1982). Digesta (ca $200 \mathrm{~g}$ ) combined from the rumens of 3 sheep grazing the same pasture as the kangaroos was introduced into the rumen of each sheep in the other room (Group S) and it contained $1.45 \times 10^{6}$ ciliates $/ \mathrm{ml}$. From samples of rumen digesta taken from the sheep just before the inoculation, it appeared that appreciable numbers of Entodinium had reestablished in 2 sheep in Group K and 3 sheep in Group S.

No ciliate protozoa from kangaroos were observed in the rumen contents of sheep in Group K 14 days after inoculation. By this time Entodinium had become established in all sheep in Group K despite precautions to isolate the sheep. Diplodiniinae became established in one of these sheep, Dasytricha in another and both of these in a third. Total concentrations of ciliates ranged from 0.04 to $5.46 \times 10^{6} / \mathrm{ml}$. The populations which became established in the sheep in Group $S$ contained Entodinium, Diplodiniinae, Isotricha and Dasytricha in similar proportions as in the inoculum and total concentrations of ciliates ranged from 0.52 to $1.96 \times 10 \% / \mathrm{ml}$. There was no effect of the form of the diet on the numbers or on the population composition of the ciliates that became established in the sheep $(P>0.05)$.

Despite the apparent similarities in the microbial ecosystems of the macropodine forestomach and the sheep rumen, neither the niches available following defaunation nor a major reduction in ciliate numbers in the sheep rumen appeared to be compatible for establishment of ciliates from the kangaroo forestomach. Competition with Entodinium seems unlikely as at least 6 of the sheep in Group K were free of rumen protozoa $18 \mathrm{~h}$ after inoculation. These observations suggest that the ciliates in the kangaroo are specific to the macropodine forestomach. The biological basis for this specificity is as yet unknown.

This work was supported in part by Australian woolgrowers throughout the International Wool Secretariat, and by the Organisation for Economic Co-operation and Development. 